\title{
Aproximación a las formas de subjetivación jurídica en mujeres víctimas de violencia doméstica*
}

\author{
Approach to Forms of Legal Subjectivity in \\ Victims Women of Domestic Violence
}

Recibido: marzo 7 de 2013 | Revisado: junio 20 de 2014 | Aceptado: junio 20 de 2014

\author{
Svenska Arensburg CAStelli ** \\ Universidad de Chile, Santiago, Chile \\ Margot Pujal Llombart \\ Universidad Autónoma de Barcelona, España
}

doi.org/10.11144/Javeriana.UPSY13-4.afsj

Para citar este artículo: Arensburg S., \& Pujal M. (2014). Aproximación a las formas de subjetivación jurídica en mujeres víctimas de violencia doméstica. Universitas Psychologica, 13(4), 1429-1440. http:// dx.doi.org/10.11144/Javeriana.UPSY13-4.afsj

* De la tesis doctoral de la autora: "Dispositivo jurídico y tramas de calificación victimal. Estudio cualitativo: víctimas de delito y ruta procesal penal en Chile" (2011).

*** Correo electrónico: sarensburg@u.uchile.cl

\begin{abstract}
RES UMEN
Con base en la investigación titulada "Dispositivo jurídico y tramas de calificación victimal. Estudio cualitativo: víctimas de delito y ruta procesal penal en Chile", en el presente artículo interesa discutir la posición que adquieren las mujeres que han experimentado violencia de pareja en su cruce con el campo jurídico, asumiendo el interés por fortalecer una perspectiva contextual, multidimensional y compleja del problema. Se parte de conceptualizar una noción de violencia en conexión con una matriz binaria sexo/género como dispositivo de poder, lo cual permite abordar el problema de la violencia de género desde la noción de violencia estructural. Conceptualización que conduce, a su vez, a analizar cómo la propia experiencia de las mujeres que denuncian ante la institucionalidad jurídica por situaciones de violencia contra ellas, es capturada por un dispositivo de persecución que tecnifica, reduce y reconduce la demanda subjetiva/política. Captura cuyo efecto final es nutrir al propio dispositivo de control de género, a través de la imposición de un ideal jurídico de víctima que termina revictimizando a las mujeres. Palabras clave

violencia de género; proceso penal; mujeres
\end{abstract}

\begin{abstract}
A B S T R A C T
Upon the basis of a previous study ${ }^{1}$, the present article will discuss the legal implications of the position acquired by women who have experienced intimate partner violence, attempting to provide a strong contextual, multidimensional, and complex view of this issue. Its starting point is the conceptualization of the notion of violence through a binary sex/gender matrix that functions as a power device, which will allows us to tackle the problem of gender violence from the notion of structural violence. In turn, this conceptualization leads us to analyze how the experience of women who denounce their partners before the legal establishment for violent acts against them is captured by a prosecution device which technifies, reduces, and redirects women's political/subjective claims. The final effect of such capture is the nourishment of the gender control device through the imposition of an ideal legal image of the victim which re-victimizes women. Keywords: gender violence; legal process; women
\end{abstract}

1 Entitled "Legal Devices and Structures of Victim Qualification. Qualitative Study: Victims of Crime and the Route of the Prosecution Process in Chile" (2011) by Svenska Arensburg. 


\section{Introducción}

\section{Derecho, violencia doméstica $y$ violencia de género}

La violencia como matriz comprensiva constituye hoy uno de los ejes centrales en el análisis de la convivencia y bienestar social, al tiempo que se presta como punto de articulación relevante para el desarrollo de políticas públicas y líneas de intervención disciplinarias. Existen diversos programas e instituciones que se ocupan del problema (Organización Mundial de la Salud, 2003).

Más particularmente, desde la Conferencia de Belém do Pará (1994), se han desarrollado instrumentos internacionales que obligan a los Estados suscriptores a la implementación de estrategias pluridimensionales e interinstitucionales para el abordaje del fenómeno tipificado como violencia de pareja o doméstica, instrumentos que proponen una comprensión del problema y una intervención sobre el mismo, desde donde se releva especialmente el valor de un modelo ecológico y de género (Secretaría de Relaciones Exteriores, 2007).

Las iniciativas y programas de intervención en este campo específico de la violencia de género se han centrado en: a) hacer públicas las situaciones de violencia, b) perseguir jurídicamente sus consecuencias y c) implementar respuestas frente a las propias mujeres a través de iniciativas de protección y recuperación. Hoy es posible aseverar que son respuestas que se han consolidado institucionalmente e internacionalmente (Larraín, Valdebenito, \& Rioseco, 2008).

En el presente artículo se dedicará, particularmente, al fenómeno construido en torno del ejercicio de la violencia doméstica, cuando las mujeres que denuncian toman contacto con las formaciones jurídico-penales y son inscritas en la posición de víctimas de delito de violencia intrafamiliar (en adelante delito constitutivo de VIF).

Cuando en los estudios realizados previamente se parte del testimonio de las propias mujeres que han experimentado una historia de maltrato por parte de sus parejas, o cuando en el operar institucional se toma contacto y se observa la relación entre un hombre y una mujer en su vínculo singular, se reconoce lo que los profesionales denominan actualmente "un régimen de violencia" (Lewin et al., 2011). Es decir, un modo de relación donde la violencia sexual/genérica constituye un ejercicio de la violencia fundamentada en la diferencia sexual, que organiza el "habitus" (Bourdieu, 1997, 2000) que hace posible sostener esa específica relación, que no será otra que una específica relación de dominación. Cuestión analizada por la tradición de estudio del fenómeno, a partir de la noción de "ciclo de la violencia" y la de "síndrome de la mujer maltratada" (Walter, 1979, entre otros).

Por lo tanto, la perspectiva del presente artículo apunta a exponer las propias formas que tiene el conocimiento de capturar el sufrimiento de mujeres maltratadas en contextos domésticos, para conectar el estudio del fenómeno con las claves de análisis del campo general de la violencia de género y sexual, desde donde reflexionar sobre el abordaje institucional del fenómeno dado el estatuto estructural que fundamenta dichas violencias.

\section{Violencia entre los géneros}

Lo que se ha llamado violencia entre los géneros cruza al menos tres grandes ejes que permiten exhibir un tipo de trama que es ante todo producida en la modernidad: 1) el campo de calificación de la violencia, 2) las relaciones de poder y 3) unos discursos sobre los géneros que se han articulado como dispositivos de dominación.

\section{Calificación de la violencia}

Desde un momento nuestras sociedades se reconocen como violentas, imponiendo formas históricas de inmunización contra ellas (Esposito, 2009). Es la Modernidad política la que alejará la violencia física de diversas esferas de la actividad humana para quedar concentrada en el Estado. A partir de ese momento, la violencia devendrá aquello ilegítimo que rompe con el ordenamiento social; por lo tanto, el ejercicio violento del Estado no será otra cosa que un poder legítimo amparado en el contrato social-legal. 
Al amparo de un Estado de derecho se dividirá la concepción de violencia. Entre una violencia legítima que ejercerá el Estado en contra de una violencia ilegal que debe ser perseguida, expulsada, exterminada, particularmente entendida como violencia que atenta la paz social, la convivencia y el contrato. A partir de ese momento, el poder transferido por el derecho y luego por la expansión de los dispositivos de control y los de seguridad se configurará una matriz tecnológica, un dispositivo socio-individual abocado a inmunizar la cultura de su propia violencia. Como consecuencia, la violencia fundacional del orden social quedará sepultada en tanto violencia para representarla como ejercicio político y de autoridad. Sin embargo, con la caída del ideal de progreso civilizatorio que trae consigo la política de exterminio en la segunda guerra mundial, se volverá necesario volver a pensar la violencia, dentro del campo de disputa sobre el sentido y comprensión de la misma cuestionando los discursos hegemónicos-modernos sobre la violencia. Autores como Benjamin (1991), Arendt (2006), Galtung (1981), Bourdieu (2000) y Žižek (2003, 2009) aportarán en ese sentido.

A partir de ahí, es el propio dominio del derecho el que debe ser pensado como parte del dominio de la violencia. Para Esposito (2009), el decir de Benjamin sería que en el funcionamiento del derecho, en lugar de ser eliminada, la violencia es asumida por el poder que la prohibiría. Para Benjamin, la violencia sería en rigor lo que configura al derecho, en un movimiento que va desde su instauración arbitraria en tanto que fuerza a su mantenimiento y conservación en tanto que poder (Galende, 2009). Y esto es sustancial por cuanto si se niega el estatuto violento de los sistemas judiciales o del derecho, "se presentan como liberados de la posibilidad de regularlos y monitorear sus potenciales excesos cuando devienen ilegítimos" (Coll-Planas, García-Romeral, Mañas, \& Navarro-Varas, 2008, p. 198).

Sobre la base de estos planteamientos, adquiere sentido considerar el surgimiento de nociones como "violencia estructural" (Galtung, 1981), "violencia simbólica" (Bourdieu, 2000) y "violencia sistémica" (Žižek, 2003, 2009), para dar cuenta del problema entre una violencia estructurante del orden que es invisibilizada versus unas violencias visibles, sociales, experimentadas como tales que solo se materializan como consecuencia de formaciones culturales específicas internas a ellas. De acuerdo con Žižek, por violencia sistémica se puede entender aquella constitutiva del orden; es una violencia no experimentada como tal: "la violencia fundacional no es percibida como tal porque es el acto que establece el orden, funda el orden y a la vez se inscribe como acto legítimo en ese orden” (Žižek, 2003, p. 303).

Tomando en consideración estos planteamientos, el autor posteriormente distinguirá entre las violencias objetivas (sistémica, estructural y simbólica) y las violencias subjetivas (emergencias sociales, individuales y colectivas). Mientras que la violencia objetiva no puede atribuirse a los individuos pues es sistémica y anónima, la subjetiva es ejercida diariamente por los actores sociales siendo fácilmente denunciada (Žižek, 2009). Desde esta perspectiva, se sostiene una noción de violencia comprometida en la conformación del orden cultural en sí mismo, sosteniendo la diferencia interna sobre las violencias.

\section{Relaciones de poder}

En ese sentido, la pregunta por las condiciones de configuración del orden es una pregunta especialmente crítica, porque muestra como las relaciones de poder trabajan para evitar interrogar la estructura de dominación, manteniendo justamente su carácter de orden. Como concibiera Foucault: "no hay ejercicio de poder sin una cierta economía de los discursos de verdad" (2001, p. 34), y es de acuerdo con esa perspectiva que el derecho se forja en instrumento y medio de un ejercicio de poder, sosteniendo ciertas relaciones de dominación en la sociedad.

Entre los abordajes de Bourdieu (2000), se muestra cómo las consecuencias fundamentales de la violencia simbólica suponen sostener determinadas relaciones de dominación. Para el autor, por violencia simbólica se entiende cuando el dominado no solo termina haciendo lo que el dominador inscribe en él -solo en la medida que este está revestido como autoridad-, sino que en el proceso de 
transformación, además, signa qué posición tiene el dominado. "La forma de la dominación goza, entonces, de un reconocimiento universal afirmado en la objetividad de las estructuras sociales. Funcionan como matrices de percepciones, pensamientos y acciones de los miembros de una sociedad, imponiéndose como trascendentes y universales respecto a la experiencia personal o particular" (Bourdieu, 2000, p. 28). En este sentido, Femenías (2008) recuerda que la dimensión simbólica inherente a las relaciones de dominación se presenta en los discursos de legitimación a través de los cuales los dominadores intentan obtener la adhesión voluntaria de los dominados. El poder simbólico constituye al mundo al enunciarlo y actúa sobre él al instituir una cierta representación de ese mundo.

\section{Dispositivo de género}

Entonces es cuando se advierte que para entender el fenómeno de violencia contra las mujeres debe suscribirse una matriz comprensiva de ciertas relaciones de dominación donde la violencia de género se ejerce y tiene efectos precisos. Si bien, y de manera dominante, se ha comprendido la violencia de género como expresión subjetiva de violencia, en el presente artículo interesa subrayar su fundamento estructural.

La utilización del concepto de género (p. ej., Fries \& Facio, 1999; Rubin, 1975; Scott, 1985) tiene como finalidad dar cuenta de una formación histórica. Siguiendo a Fries y Facio: "Las teorías de género sostienen que el sistema sexo/género atribuye características, aptitudes y actitudes como masculinas y femeninas otorgándole una jerarquía y un privilegio a las definidas como masculinas, así como valorando a éstas últimas como paradigmas del ideal de ser humano" (pp. 34-35). Para las autoras, y de acuerdo con Saffioti (1992), "las personas al nacer son transformadas a través de las relaciones de género en mujeres y hombres, de tal manera que cada una de esas categorías-identidades excluye a la otra. Así el género apela a la transformación de ciertos individuos en mujeres y los otros en hombres, ese proceso es obra de las relaciones de género y no de la biología o la anatomía. Desde esta perspectiva somos prisioneros del género" (p. 36). Es así como las perspectivas teóricas sobre el sistema sexo/género permiten visibilizar los efectos de la construcción social de los géneros.

\section{Patriarcado como ordenamiento histórico/cultural}

Patriarcado corresponde a una noción que encontramos en Engels o en Weber, quienes lo caracterizan como el sistema de dominación histórico más antiguo, aunque su desarrollo específico como sistema de dominación del hombre sobre la mujer es inaugurado por teóricas feministas (Millet, 1973; Rubin, 1975). En este sentido, Fries y Facio (1999) dan por acertada a Sau (1981) cuando refiere que patriarcado significa la toma de poder histórica de los hombres sobre las mujeres, aduciendo razones de inferioridad biológica y tiene su origen en la jefatura que el padre ejercía en la familia (Fries \& Facio, 1999, p. 45).

Es entonces posible aseverar que la maniobra histórica, eje de servidumbre de lo femenino sobre lo masculino y de la subordinación de las mujeres, nace de los sistemas patriarcales. Según Rich (1978) consiste en el poder de los padres, un sistema familiar, ideológico y político que luego determinará el papel de hombres y mujeres. Es decir, la apropiación de la capacidad sexual y reproductiva de las mujeres por parte de los hombres (Rodríguez, 1999, pp. 58-59), a través de la división sexual del trabajo, estrechamente vinculada al "trafico o intercambio de mujeres" entre los hombres de familias distintas, que analiza Rubin (1975), el cual está mediatizado por el tabú del incesto y de la homosexualidad y la obligación de la heterosexualidad. Por lo anterior, para Pateman (1995) el lugar del patriarcado se sitúa en la idealización de la figura paterna que viene a devaluar a las mujeres, pero se yergue como política de los iguales, escondiendo el contrato sexual sobre las mujeres.

Una de las líneas conceptuales que aquí interesan es la situación en lo que Amigot y Pujal (2009), y de acuerdo con los trabajos de Butler (2001a), sostienen sobre género y sexualidad bajo la noción de dispositivo de poder, que por un lado produce subjetividades y cuerpos vinculados a él y, por 
otro, ocasiona y regula las relaciones estratégicas de poder entre varones y mujeres. En este sentido, entender el género como dispositivo de poder permite visibilizar los procesos de subordinación de las mujeres al género femenino y sus efectos ante la forma tradicional de patologizar el cuerpo de las mujeres: en el sentido de que el cuerpo de la mujer a lo largo de la historia se ha sobresexualizado y patologizado, amoldándolo para cumplir la función social asignada de reproducción y cuidado de la familia (Foucault, 2007).

En síntesis, tal como señalan diversas autoras, entre las que destacan Kristeva (1995), Butler (2001a) e Izquierdo (2010), el sujeto de la acción resultante del régimen abusivo entre los géneros sexuados es el sexismo y el patriarcado. En este orden de análisis, es que nos parece que las teorías feministas aportan con nuevas reflexiones, al generar una posición colectiva desde donde resistir los efectos del sistema sexo/género.

Por ello, el planteamiento de una epistemología feminista (p. ej., Fox Keller, 1978; Haraway, 1991; Harding, 1996) que supone una configuración conceptual del género específica y situada, permite sostener que el dispositivo de violencia de género no puede ser criticado con sus mismas categorías, pues estas han hegemonizado lo que es posible pensar y lo que no. Inscribirse en esta comprensión permite determinar cómo nuestra cultura está atravesada por la violencia contra las mujeres y por lo tanto violencia sexual y de género es una expresión histórica arraigada en las propias formaciones culturales.

Por lo tanto, cuando hablamos de "violencia de género", siguiendo la definición que hiciera la ONU en la Asamblea General de 1993, será acertado definirla como "cualquier acto o intención que origina daño o sufrimiento físico, sexual o psicológico a las mujeres, incluyendo las amenazas de dichos actos, la coerción o privación arbitraria de libertad, ya sea en la vida pública o privada". Desde un punto de vista feminista, por violencia sexista se entiende a las formas de trasgresión por medio de castigos y disciplinamientos, "mecanismos que perpetúan la posición subordinada de las mujeres en el orden patriarcal que aún predomina en nuestra cultura" (Montaño, 2007, p. 15). En este orden, reconoce- mos, como parte de las definiciones de violencia contra las mujeres, la noción de violencia doméstica publicada en España en la Ley Orgánica 1/2004 de 28 de diciembre, de Medidas de Protección Integral contra la Violencia de Género: "violencia como manifestación de la discriminación, la situación de desigualdad y las relaciones de poder de los hombres sobre las mujeres, que se ejerce sobre éstas por parte de quienes sean o hayan sido sus cónyuges o de quienes estén o hayan estado ligadas a ellas por relaciones similares de afectividad, aún sin convivencia" (Ley Orgánica 1/2004).

A través de este recorrido, nos interesa expresar cómo los excesos visibles, sociales, relacionales y subjetivos a nivel de los conflictos de género, no serían explicables como expresiones de locura, patología individual o barbarie contracultural, y por lo tanto no solo comprensibles y condenables como violación al contrato social ni como mera violencia subjetiva, sino resultan ser efectos de una violencia estructural naturalizada bajo las formas del sistema sexo/género, violencia que se despliega del propio tejido articulado por el patriarcado para sostenerse como tal. Esto quiere decir que la violencia doméstica no puede disociarse de la comprensión del género como dispositivo de poder; en otras palabras, la violencia doméstica es una expresión dentro de la violencia de género que estructura las relaciones de dominio en nuestras sociedades y, por lo tanto, no puede analizarse con independencia de una concepción de relación de poder donde toma lugar y sentido una estructura de género patriarcal. En este sentido, surge el desafío de detectar formas de normalizar o estandarizar los efectos de la violencia de género, por ejemplo, prácticas que reducen la explicación a motivos psicológicos o tecnificaciones jurídicas.

\section{El dispositivo de género en el derecho procesal penal: revictimización de las víctimas}

El abordaje público del fenómeno de violencia doméstica tiene un recorrido de casi dos décadas, caracterizándose por su heterogeneidad. En Chile, es abordado bajo la nominación de violencia intra- 
familiar, nombre impreciso, pues a pesar que en él se incluya mayoritariamente el maltrato, abusos y agresiones contra mujeres en el matrimonio y la relación de pareja, también supone incorporar otros fenómenos abusivos que se dan al interior de la institución familiar, teniendo como consecuencia, políticas vagas e imprecisas en el abordaje del problema. En España, se habla de violencia doméstica, violencia en la pareja, etc. En los estudios sobre violencia doméstica, frecuentemente se hace visible una cierta incomodidad de la propia institución familiar expuesta públicamente, incomodidad del campo jurídico al verse enfrentado con un fenómeno recientemente visto como privado, incomodidad social frente a la denuncia de las mujeres que se presentan o como víctimas o como insaciables en su demanda.

Consideramos que una de las fuentes de estas dificultades es la ausencia de un enfoque complejo que incorpore la especificidad de la violencia contra las mujeres, en este caso particular para el contexto familiar y de pareja. Tal como se pudo analizar en la investigación realizada (Arensburg, 2011) en Chile, y tal como lo confirman Larraín et al. (2008), la nueva Ley de Violencia Intrafamiliar № 20.066 vigente constituye un avance sustancial en la respuesta a la violencia contra la mujer; sin embargo, continúa llamándose "Ley sobre Violencia Intrafamiliar", lo que significa que no se incorpora la perspectiva de género como un factor relevante en el abordaje de la violencia hacia la mujer (Arensburg, 2011, p. 424).

En el mismo orden argumentativo, Larraín et al. (2008) señalan que las respuestas del Estado orientadas a la violencia de género que se han implementado a la fecha se han concentrado principalmente en la respuesta a las víctimas y sanción a los agresores, pasando a segundo plano la prevención de la violencia. Para estas autoras, la tendencia a la excesiva penalización y regulación legal de fenómenos sociales, muy propia de algunos países como Chile, en el caso de la violencia contra las mujeres, si bien es entendible al enfrentar la violencia de género, no puede ser la única respuesta (Arensburg, 2011, p. 249).

Diversos estudios constatan la cantidad de mujeres que son víctimas de actos de violencia sexista en la pareja (Centro de Análisis de Políticas Públicas de la Universidad de Chile, 2002; Instituto Nacional de las Mujeres, 2006; Montaño, 2007; Marchant \& Soto, 2011; Sepúlveda, 2004). Un eje dominante para suscribir la sanción de la violencia contra las mujeres fue recurrir a la judicialización de tal violencia. Bovino (1997), de acuerdo con Smaus (1992), observa que el recurrir al derecho penal es un medio a través del cual el Estado se hace parte del conflicto social, puesto que determinados temas morales se hacen públicos solo cuando están inscritos en un trasfondo penal, es decir, se está apelando a la función simbólica del derecho penal. Lo que conduce a hacer "reconocibles como problemas sociales ciertas situaciones padecidas por las mujeres, por el hecho de ocupar el lugar de mujeres en la sociedad" (p. 8). Como señala un fiscal especializado en este ámbito "judicializando los casos de VIF, publicitas el hecho, lo sacas del ámbito de lo privado, lo conviertes en público, con eso puedes tratar de evitar que se agraven los casos, no siempre lo logras, pero creo que para un grupo eso ya de por si sirve" (Arensburg, 2011, Entrevistado 14, fiscal especializada, VIF).

Sin embargo, para el mundo judicial el encuentro con dichas mujeres no resulta ser una tarea fácil. En esa dirección van las palabras de un fiscal que aborda los casos VIF en Santiago de Chile: "El tipo de víctima lo hace complejo (...). Entonces si no se entiende el fenómeno produce mucha desazón" (Arensburg, 2011, p. 434, Entrevistado 17, fiscal).

Esta forma de expresión habla más bien del propio lugar que toma la violencia para la subjetividad de las mujeres de estos casos, y en especial expresa las severas formas de resistencia y conflicto que enfrentan en relación con reconocer, terminar y denunciar la violencia doméstica de la que son víctimas. Tal como advierte una entrevistada: "En un régimen de violencia, se trata de sostenerse en la identidad que el otro da, esa posición del agredido es una posición que se toma, es respecto a lo que el agresor dice que es, lo que ellos dicen de ellas, como las definen. Ella adquiere su identidad sobre lo que él dice, el efecto de su palabra es muy fuerte en ellas" (Lewin en Arensburg, 2011, p. 241). En este sentido, estamos con lo señalado por Crempien (2005): "La 
revictimización nos parece especialmente relevante en el trauma relacional, pues nos ayuda a entender mejor dos fenómenos: la dificultad de las mujeres que sufren abusos de terminar estas relaciones, tendencia a permanecer y volver con sus parejas a pesar de la violencia" (Crempien, 2005, p. 93).

Sin embargo, a pesar de lo que podría leerse como un avance a este respecto, se han planteado diversas críticas respecto al modo como las formaciones judiciales enfrentan el problema de los delitos cometidos en el interior de relaciones de parejas heterosexuales. Las herramientas que disponen las formaciones jurídicas en la práctica del operar judicial no permiten enfrentar las contradicciones que emanan del propio fenómeno relacional, apareciendo como situaciones que no tienen explicación para el derecho, generando conflictos en su quehacer. En la medida en que las mujeres en posición de víctimas están vinculadas al imputado, sus intereses se ven socavados y tensionados por esa relación de lealtad al imputado, posición que se resiste a la oposición dicotómica entre la figura de la víctima y la del victimario: "(...) son las víctimas por así decir medio atípicas. Una víctima que pueda estar ligada por razones de parentesco, de relaciones con su victimario y que por lo tanto, está en constante tensión entre lo que quiere hacer, lo que necesita y lo que debe hacer frente al proceso penal, y por lo tanto tiene necesidades mucho más específicas..." (Arensburg, 2011, p. 435, Entrevistado 2, abogada docente, VIF).

El tipo de respuesta penal a las mujeres en calidad de víctimas de delito está lejos de responder las demandas de las propias mujeres, pues la propia formación judicial está teñida de los dispositivos patriarcales que reifican los principios jerárquicos de la división genérica, desconociendo las bases culturales que hacen posible la reproducción de la violencia de género. En Casas y Mera esto se demuestra abundantemente:

La intervención judicial está prejuiciada por la construcción de estereotipos sobre la víctima, la clase, el origen étnico, la edad y el sexo. En el caso de las mujeres, la víctima es percibida como legítima y su denuncia es real y por consiguiente merece protec- ción en mayor medida, mientras más se acerque a los patrones socioculturales de superioridad moral que se le atribuye a las mujeres. (Casas \& Mera, 2004, p. 6)

Por lo tanto, en el cruce entre la experiencia de las mujeres y su contacto con el operar judicial uno de los efectos más sensibles analizados es la revictimización de las mujeres.

En este orden de sentido, Coll-Planas et al. (2008) sostienen que la concepción de las mujeres solamente como víctimas pasivas se traduce en la atribución de un papel que niega a las mujeres la posibilidad de actuar conscientemente para acabar con su subordinación, dejándoles como única salida pedir ayuda a otros. Al respecto, sería consecuente para los autores, siguiendo a Nussbaum (2001), que el sentimiento de compasión surja como consecuencia de otros requisitos: "cuando la persona que observa considera que la que sufre no se merece o no es responsable de su sufrimiento -en el caso contrario, no sentirá compasión, sino que le culpará de su situación-" (p. 201).

Tal como pudimos tomar nota en la investigación realizada (Arensburg, 2011), las prácticas judiciales centradas en una concepción ideal y universal de víctima de delito construyen un territorio donde los testimonios de las propias mujeres en posición de "víctimas VIF" transcurre por unos estereotipos que impiden escuchar la experiencia que se denuncia, confirmando los análisis anteriores a nivel internacional (Montoya, 2000; Stanko, 1981; Stewart, Dobbin, \& Gatowski, 1996).

Las consecuencias más delicadas que devienen de estas prácticas pueden entenderse como un dispositivo que subjetiva a las mujeres psicologizando su padecer y precarizando su campo de acción y subversión política. La construcción de una subjetividad precaria en las mujeres, en virtud del dispositivo de género reificado en las formaciones judiciales, tiene múltiples efectos sociales, analizados en otros estudios (Izquierdo, 1998). Para Hercovich (1992), por ejemplo, la victimización de que son objeto las mujeres frente a la institucionalidad responde al mismo dispositivo, que tiene como resultado vitalizar el paradigma 
victimizador, el cual reduce la violencia a un problema de patologías mentales, anormalidades del victimario y predisposiciones y daños mentales de la víctima, dejando fuera del problema el análisis de las relaciones de dominación que suponen las prácticas de sometimiento de las mujeres. Situar a la mujer como víctima inocente, objeto, pasiva, tiene como consecuencia situar a las mujeres fuera del campo de los sujetos, sujeto de negociación, de autonomía, de-subjetiva su testimonio, relegando su condición a la impotencia (Arensburg, 2011, p. 77). En estos términos y de acuerdo con Bodelón y Bergalli (1993): "el proceso de criminalización de la violencia por género, hizo que todos los elementos simbólicos que podían encontrarse expresados en la denuncia de la violencia se vieran relegados a la expresión de dicha violencia mediante el sistema penal y tiene la perversa consecuencia respecto a las mujeres, de convertirlas en víctimas (frágiles, pasivas, objetos de otro) sin aportar en nada a su reconstrucción subjetiva” (p. 21).

A partir de lo anterior, Bovino (1997) sostiene que reconocer el valor simbólico del Estado en el proceso de hacer público un problema social supone asumir el desafío de ampliar su respuesta, particularmente porque esta no aporta al reconocimiento del interés de la víctima como sujeto de derecho ni menos a la reparación de la misma (p. 15). La contradicción está servida: "Si uno la trata como víctima ella no toma en sus manos su proceso. Si no la tratas como sujeto de derecho y decides por ella no produces ningún cambio, ese es el enganche, que sea el ritmo de ella porque si no no hay cambio" (Arensburg, 2011, p. 446, Entrevistado 18).

El problema que plantea restringir la situación de las mujeres al perfil de victimicidad es que alimenta una posición de víctima como nicho identitario, lo que acarrea como consecuencia ir reforzando y alimentando especialmente el dispositivo de género. Esta operación releva la posición estándar de víctima para adecuarla a los requisitos de las prácticas procesales, sin reconocer, por un lado, a) la complejidad de la de-subjetivación que exhiben las personas sobrevivientes de relaciones violentas $y$, por otro, b) debilita las voces que pretenden le- vantar y promover el discurso de las sobrevivientes como actrices sociales.

En este sentido, tal y como señalan Larraín et al. (2008), parece que las instituciones públicas no han identificado del todo - por diversas razones- el potencial que implica la participación real de las víctimas y de los organismos de la sociedad civil que se han especializado en la materia.

\section{De víctima a sujeto vulnerado y de derecho}

En estos términos, las prácticas que envuelven y fijan a las formaciones judiciales ocupan un lugar especialmente crítico respecto al tratamiento de la violencia doméstica. Si bien la visibilizan, al concentrarse en la punición del culpable y la hiperprotección de la víctima, acallan la demanda de las propias mujeres. Una demanda compleja por cuanto asumiendo la necesidad de soporte es a la vez el pronunciamiento de las mujeres como sobrevivientes a los excesos de la dominación de género. Entonces, el tipo de tratamiento procesal penal opera como una nueva experiencia de invalidación y de violencia contra la demanda de las mujeres. Un campo potencial entonces será pensar las formas de recuperación, desde la propia demanda de las mujeres en su relación con la violencia, una violencia nacida de la propia estructuración cultural de género.

Desde nuestro punto de vista, las formas que tomen las estrategias de intervención y aproximación al padecimiento de las propias mujeres han de ser capaces de incidir, de manera sustantiva, en un desplazamiento de su posición en el régimen de violencia. A partir de la comprensión de su "no lugar" como consecuencia de la experiencia de violencia y su falta de posición subjetiva y adaptación continua al otro, se plantea la necesidad de un movimiento crítico de re-subjetivación, un movimiento que no puede hacer como individuo, pero que tiene que hacer por sí misma, en su singularidad. Esa delicada frontera opera como un acontecimiento de encuentro entre ese acto de palabra sin sujeto y un tercero que haga posible que esas mujeres se miren y se vean, en términos de reconocimiento y 
afirmación, mediante una relación de sujeto a sujeto o relación de amor y no de abuso (Benjamin, 1997). A través de este proceso de encuentro/reconocimiento/afirmación, el momento contingente de re-subjetivación puede moverse inicialmente hacia la posición de "víctima" y también, aunque en general a posteriori, hacia una posición de autonomía.

La promoción de la condición de víctima a sujeto vulnerado como transición hacia la posición de sujeto de derecho, se entenderá como labor de acompañamiento y soporte, personalizando el sentido de su trayectoria. Ruta de la que dan cuenta las voces de algunas profesionales: "Salir de la violencia es un cambio de la posición subjetiva, posición de sometimiento, posición y condición de esa mujer" (Arensburg, 2011, Entrevistada 18, abogada ONG "Humanas"). O bien: "Cuando tu te contactas con tu propio poder, con tu fortaleza, te juntas con otra eso te da una visión de ti y tu problema distinto, a estar aislada, sola. Las personas que viven violencia están distanciadas de su propia experiencia de violencia, contactarse con eso lleva bastante tiempo" (Arensburg, 2011, Entrevistada 20, profesional RED CH.V.D.S).

Podríamos resumir que dicha trayectoria recorre un camino que va desde: a) la de-subjetivación que ha producido un régimen de violencia sexual/ genérica, b) hacia el asumir el lugar de víctima en ese régimen de violencia (que puede o no coincidir con la interpelación como víctima del proceso judicial, siendo lo más probable que no coincida), para finalmente llegar a un destino de c) reconocerse como actriz y partícipe de la construcción de su vida, "hacerse cargo de su vida", un hacerse cargo soportado por otro/otra como acompañamiento en la recuperación de sí (Arensburg, 2011, p. 451).

Es así como las salidas propuestas desde la teoría del derecho, que adopta una perspectiva de género en términos de justicia y derecho no androcéntricos con el fin de desembarazarse de los fundamentos de la violencia de género, van en la línea de una articulación situada entre la ética de la justicia y la ética del cuidado (Bodelon, 2010), como vía posible de eje articulador de las líneas que permitan la re-subjetivación de las mujeres denunciantes de violencia doméstica. Esto significa asumir, como punto de partida, el hecho que las mujeres están desarrollando sus derechos en estructuras todavía con un marcado sesgo de género (sobrecargas, exclusiones, etc.), a pesar de la retórica igualitarista dominante en sociedades occidentalizadas, por lo que es necesario complejizar el concepto de justicia. Como plantea López de la Vega (2008), es deseable teóricamente en el derecho un equilibrio entre justicia y cuidado, además de una práctica jurídica en la que el género no sea criterio a la hora de atribuir responsabilidades. A su vez, también es necesario situar histórica y socialmente el concepto de cuidado sin esencializarlo o asociarlo a las mujeres, puesto que en sí mismo tiene todavía que ver con relaciones de poder y desigualdad. En este sentido, para esta articulación entre la justicia y la ética, el sujeto de derecho ya no sería ni esa concepción abstracta -universal-normativa (bien jurídico) de sujeto general, ni ciertamente esa presencia empírica-individual-descontextualizada-autosuficientede la victimología positivista.

\section{Reflexiones finales}

A partir del recorrido antes propuesto, es posible sostener que la violencia de género en su dimensión estructural atraviesa las prácticas jurídicas tanto como a las vías vinculares que hacen posible las relaciones de dominación y abuso en el plano doméstico. Sin embargo, y como consecuencia de la forma en que se ha configurado institucionalmente el dispositivo judicial, es necesario puntualizar algunos elementos centrales respecto de sus consecuencias específicas. Por un lado, las herramientas vigentes que disponen las formaciones jurídicas en la práctica del operar judicial no permiten enfrentar las contradicciones que emanan del propio fenómeno relacional que traen las mujeres, obturando la escucha de la dinámica producida por la dominación de género. Por otro lado, el problema que plantea restringir la situación de las mujeres al perfil de victimicidad es el de alimentar una posición de víctima como nicho identitario, reificando tal lugar como lugar de ser del sujeto mujer, lo que acarrea como consecuencia ir reforzando el dispositivo de género distanciándola del paradigma de sujeto de derechos. 
A partir de lo anterior, los procesos de subjetivación que allí operan como estrategias de reconocimiento y validación de la denuncia de las mujeres toman la forma de psicologizar la comprensión de su demanda, estandarizar sus dichos, silenciar las condiciones de surgimiento del régimen de violencia, reducir su posición a la condición de víctimas y/o despolitizar la posición de las propias mujeres. Desde nuestro punto de vista estas formaciones invisibilizan el estatuto estructural que sostienen tales relaciones de dominación, haciendo de las formaciones judiciales una expresión y no una excepción de ellas.

Entonces, una perspectiva de género, para considerarse elemento sustantivo en el análisis de los fenómenos de violencia, debería constituirse en una mirada transversal, interdisciplinar, multidimensional y sociohistórica, por lo que el desafío sería introducirla en los múltiples ámbitos de producción cultural, discursiva y de intervención social e institucional. Y, especialmente, debiera partir por considerarse una forma de análisis de las relaciones de dominación sustentadas culturalmente. Desafío del que seguro emergerán múltiples resistencias. Se ha visto una de las más significativas: cuando el hecho de exigir punición para el abuso contra las mujeres, resultó, por un lado, en un grito sordo para la emancipación de las propias mujeres y, por otro lado, en un instrumento fértil para alimentar dispositivos punitivos del Estado que individualizan, encierran y patologizan el enfrentamiento del fenómeno de violencia doméstica. Queda pendiente, pues, la producción de conocimiento en y más allá del ámbito de las formaciones jurídicas, orientadas a la emancipación de las mujeres y a la liberación del dispositivo de control binario de género tanto por parte de mujeres como de hombres, en sus múltiples contextos, en pro de la construcción social de un saber ética y políticamente orientado a la igualdad y a la diversidad al mismo tiempo.

Esta forma de transformación reclama un nivel reflexivo por parte de la episteme y práctica del derecho (procesal penal), para que pueda integrar una concepción compleja de sujeto de derechos, que supone formas de subjetividad diversas y atravesadas culturalmente. Las concepciones de género y sus efectos prácticos solo terminan por hegemonizar la producción cultural de mentalidades e identidades que alimentan el control social en detrimento de la libertad y la igualdad de los sujetos de derechos.

\section{Referencias}

Amigot, P., \& Pujal, M. (2009). Una lectura del género como dispositivo de poder. Sociológica, 24(70), 115-152.

Arendt, H. (2006). Sobre la violencia. Madrid: Alianza Editorial.

Arensburg, S. (2011). Dispositivo jurídico y tramas de calificación victimal. Estudio cualitativo: víctimas de delito y ruta procesal penal en Chile (Tesis doctoral). Universitat Autònoma de Barcelona, España.

Benjamin, J. (1996). Los lazos del amor. Psicoanálisis, feminismo y el problema de la dominación. Buenos Aires: Paidós.

Benjamin, W. (1991). Para una crítica de la violencia y otros ensayos. Madrid: Taurus.

Bergalli, R., \& Bodelón, E. (1993). La cuestión de las mujeres y el derecho penal simbólico. "Nullum Crimen”, 2, Santa Cruz de la Sierra (Bolivia), Ed. Nueva Generación/Universitaria.

Bodelón, E. (2010). Derecho y justicia no androcéntricos [Monográfico: Desigualdades de género en "tiempo de igualdad"]. Quaderns de Psicología, 12(2), 183-193.

Bonino, L. (2003). Los varones ante el problema de la igualdad con las mujeres. En C. Lomas (Ed.), iTodos los hombres son iguales? Identidad masculina y cambios sociales (pp. 105-144). Barcelona: Paidós.

Bourdieu, P. (1997). Razones prácticas. Sobre la teoría de la acción. Barcelona: Anagrama.

Bourdieu, P. (2000). Dominación masculina. Barcelona: Anagrama.

Bovino, A. (1997). Delitos sexuales y feminismo legal: (algunas) mujeres al borde de un ataque de nervios. Revista Electrónica de la Asociación de Ciencias Penales, 9(14).

Butler, J. (2001a). Género en disputa. México: Paidós.

Butler, J. (2001b). Mecanismos psíquicos del poder. Barcelona: Cátedra.

Casas, L., \& Mera, A. (2004). Violencia de género y reforma procesal penal chilena. Cuadernos de 
Análisis Jurídico. Serie de Publicaciones Especiales, 16, 194-196.

Centro de Análisis de Políticas Públicas de la Universidad de Chile. (2002). Detección y análisis de la violencia intrafamiliar en la región metropolitana y la Araucanía (Documento de Trabajo № 121). Santiago: Servicio Nacional de la Mujer, Gobierno de Chile.

Coll-Planas, G., García-Romeral, G., Mañas, C., \& Navarro-Varas, L. (2008). Cuestiones sin resolver en la ley integral de medidas contra la violencia de género: las distinciones entre sexo y género, y entre violencia y agresión. Papers, 87, 187-204.

Corte Interamericana de Derechos Humanos. (2007). Acceso a la justicia para las mujeres víctimas de violencia en las Américas. Washington, DC: Secretaría General de la OEA.

Crempien, C. (2005). El trauma relacional de la pareja en la pareja. Impacto y consecuencias. En La violencia en la familia, escuela y sociedad. Sentidos consecuencias y estrategias de intervención (pp. 85 99). Santiago: Universidad Internacional SEK.

España, Ley Orgánica 1/2004. Medidas de Protección Integral contra la Violencia de Género. Diciembre 28 de 2004. BOE n. -913.

Esposito, R. (5 de marzo, 2009). Comunidad y violencia. Conferencia dictada en el Círculo de Bellas Artes, Madrid.

Femenías, M. L. (2008). Violencia de sexo-género: el espesor de la trama. En P. Laurenzo, M. L. Maqueda \& A. Rubio (Coords.), Género, violencia y derecho (pp. 61-88). Valencia: Tirant lo Blanch.

Foucault, M. (2001). El sujeto y el poder. En H. Dreyfus \& P. Rabinow (Eds.), Michel Foucault: más allá del estructuralismo y la hermenéutica (pp. 241-259). Buenos Aires: Nueva Visión.

Foucault, M. (2007). Historia de la sexualidad (Vol. 1). La voluntad de saber. Buenos Aires: Siglo XXI.

Fox Keller, E. (1978). Gender and science. Psychoanalysis and Contemporary Thougth, 1(3), 409-433.

Fries, L., \& Facio, A. (Eds.). (1999). Género y derecho. Santiago de Chile: LOM Ediciones.

Galende, F. (2009). Walter Benjamin y la destrucción. Santiago de Chile: LOM Ediciones.

Galtung, J. (1981). Contribución específica de la irenología al estudio de la violencia: tipologías. En J. -M.
Domenach et al. (Eds.), La violencia y sus causas (pp. 91-106). París: Unesco.

Haraway, D. (1991). Simians, cyborg, and women: The reinventation of nature. New York: Routledge.

Harding, S. (1996). Ciencia y feminismo. Madrid: Morata.

Hercovich, I. (1992). De la opción sexo o muerte a la transacción sexo por vida. En A. M. Fernández (Comp.), Las mujeres en la imaginación colectiva (pp. 63-83). Barcelona: Paidós.

Izquierdo, M. J. (1998). Los órdenes de la violencia: especie, sexo y género. En V. Fisas (Ed.), El sexo de la violencia: género y cultura de la violencia (pp. 61-91). Barcelona: Icaria.

Izquierdo, M. J. (2010). Las dos caras de la desigualdad entre mujeres y hombres: explotación económica y libidinal. Quaderns de Psicologia, 12(2), 117-129.

Kristeva, J. (1995). Tiempo de mujeres. Las nuevas enfermedades del alma. Madrid: Cátedra.

Larraín, S., Valdebenito, L., \& Rioseco, L. (2008). La situación de violencia contra las mujeres en Chile. Legislación y políticas públicas. Santiago de Chile: Unesco. http://portal.unesco.org/geography/es/ files/11343/12436345861Resumen_ejecutivo_del_ estudio_la_situacion_de_violencia_contra_las_ mujeres_en_Chile._Legislacion_y_politicas_publicas.pdf/Resumen.pdf

Lewin, E., Berlagoscky, F., Jordán, S., Porta, M., Gifreu, M., \& Jeftanovic, A. (2011, 17 de julio). Análisis sobre los testimonios de mujeres que han salido de relaciones violentas. Después de la violencia... palabras en espera, 8-20. Recuperado de http:// www.despuesdelaviolencia.net/descargas/analisis_ testimonio_mujeres.pdf

López de la Vega, M. T. (2008). Justicia y cuidado. En A. Puleo (Coord.), El reto de la igualdad de género (pp. 238-253). Madrid: Biblioteca Nueva.

Marchant, J. P., \& Soto, E. (2011). Reparación psicosocial en mujeres víctimas de violencia de pareja. Cómo se trabaja en las Casas de Acogida chilenas (Memoria para optar al título de psicólogo). Universidad de Chile, Santiago, Chile.

Millet, K. (1973). Política sexual. México: Aguilar. México, Instituto Nacional de las Mujeres. (2006). Violencia sexual contra las mujeres en el seno de la pareja conviviente. México: Autor. Recuperado 
de http://cedoc.inmujeres.gob.mx/documentos_ download/100790.pdf

México, Secretaría de Relaciones Exteriores. (2007). Convención interamericana para prevenir, sancionar y erradicar la violencia contra la mujer o Convención de Belém do Pará y su estatuto de mecanismo de seguimiento. México: SER/UNIFEM/PNUD. Recuperado de http://www.observatoriominerva. gob.mx/documentos/BelemDoParaagt07.pdf

Montaño, S. (2007). iNi una más! El derecho a vivir una vida libre de violencia en América Latina y el Caribe (Informe LC/L.2808). Santiago de Chile: CEPAL.

Montoya, Y. (2000) Ejercicio Público de la Acción Penal: la Tutela Procesal de la Víctima en los Delitos Sexuales, en Problemas Actuales de la Administración de Justicia en los Delitos Sexuales, Defensoría del Pueblo, Lima, pp. 249-269.

Nussbaum, M. (2001). Upheavals of thought: the intelligence of emotions. Cambridge NY: Cambridge University Press.

Organización Mundial de la Salud. (2003). Informe mundial sobre la violencia y la salud. Washington, DC: Organización Panamericana de la Salud, Oficina Regional para las Américas.

Pateman, C. (1995). El contrato sexual. México: Anthropos.

Rich, A. (1999). La heterosexualidad obligatoria y la existencia lesbiana. En M. Navarro \& C. Stimpson
(Eds.), Sexualidad, género y roles sexuales (pp. 159211). Buenos Aires: Fondo de Cultura Económica.

Rodríguez, R. M. (1999). Foucault y la genealogía de los sexos. México: Anthropos.

Rubin, G. (1975). El tráfico de mujeres: notas sobre la "economía política" del sexo. Nueva Antropología, 7(30), 95-145.

Sepúlveda, J. (2004). Estudio de los factores que favorecen la continuidad en el maltrato de la mujer (Tesis doctoral). Universitat de València, España.

Scott, J. (1986). Gender: A useful category of historical analysis. The American Historical Review, 91(5), 1053-1075.

Stanko, E. (1982). The impact of victim assessment on prosecutors' screening decisions: The case of the New York County District Attorney's Office. Law and Society Review, 16(2), 225-239.

Stewart, M., Dobbin, S., \& Gatowski, S. (1996). "Real rapes" and "real victims": The shared reliance on common cultural definitions of rape. Feminist Legal Studies, 4(2), 159-177.

Walter, L. (1979). The battered women. New York: Harper \& Row.

Žižek, S. (2003). Las metástasis del Goce. Seis ensayos sobre la mujer y la causalidad. Buenos Aires: Paidós.

Žižek, S. (2009). Sobre la violencia. Seis reflexiones marginales. Buenos Aires: Paidós. 